\title{
Combined screening for early and late pre-eclampsia and intrauterine growth restriction by maternal history, uterine artery Doppler, mean arterial pressure and biochemical markers
}

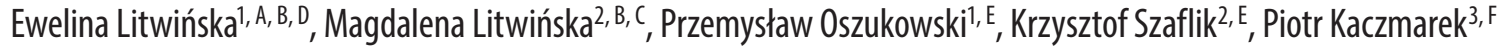 \\ ${ }^{1}$ Perinatology and Gynecology Department, Polish Mother's Memorial Hospital Research Institute, Łódź, Poland \\ ${ }^{2}$ Department of Gynecology, Fertility and Fetal Therapy, Polish Mother's Memorial Hospital Research Institute, tódź, Poland \\ ${ }^{3}$ Department of Operative Gynecology and Oncological Gynecology, Polish Mother's Memorial Hospital Research Institute, Łódź, Poland \\ A - research concept and design; $\mathrm{B}$ - collection and/or assembly of data; $\mathrm{C}$ - data analysis and interpretation; \\ $D$ - writing the article; $E$ - critical revision of the article; $F$ - final approval of article
}

Address for correspondence

Ewelina Litwinska

E-mail: ewelina.litwinska@gmail.com

Funding sources

None declared

Conflict of interest

None declared

Received on 0ctober 14, 2015

Revised on December 06, 2015

Accepted on March 16, 2016

DOI

10.17219/acem/62214

\section{Copyright}

Copyright by Author(s)

This is an article distributed under the terms of the

Creative Commons Attribution Non-Commercial License

(http://creativecommons.org/licenses/by-nc-nd/4.0/)

\begin{abstract}
Background. Pre-eclampsia is a systemic disease connected with high maternal and fetal morbidity and mortality. Despite significant progress achieved in perinatal medicine, pre-eclampsia is still one of the most significant current problems in obstetrics.
\end{abstract}

Objectives. The aim of the study was to establish diagnostic algorithms for early and late pre-eclampsia (PE) and intrauterine growth restriction (IUGR).

Material and methods. A total of 320 pregnant women between $11+0$ and $13+6$ weeks of gestation were recruited for a case-control study. The study group consisted of 22 patients with early PE, 29 patients with late PE and 269 unaffected controls. The following parameters were recorded: maternal history, mean arterial pressure (MAP), mean uterine artery pulsatility index (UtA-PI), and the concentrations of placental growth factor (PIGF), pregnancy-associated plasma protein A (PAPP-A) and free beta-human chorionic gonadotropin (free $\beta-h C G)$.

Results. A multivariable stepwise logistic regression analysis indicated that the best screening model for the prediction of early PE is based on a combined analysis of maternal risk factors, UtA-PI and PIGF levels (sensitivity: 91\%; specificity: 84\%). The best screening model for the prediction of late PE is based on a combined analysis of maternal risk factors, UtA-Pl and MAP (sensitivity: 85\%; specificity: 83\%). The most effective screening model for the prediction of IUGR is based on a combined analysis of maternal risk factors, UtA-PI and PIGF concentrations (sensitivity: 91\%; specificity: 83\%).

Conclusions. The integrated model of screening established in this study can be a valuable method to identify patients at increased risk of developing pre-eclampsia and related complications. The ability to predict the occurrence of pre-eclampsia in early pregnancy would enable maternal and fetal morbidity to be reduced through the introduction of strict obstetric surveillance as well as planned delivery in a reference center.

Key words: pre-eclampsia, placental growth factor, diagnostic algorithms 
Pre-eclampsia (PE) is a disease characterized by high maternal and fetal morbidity and mortality. As many as $3 \%$ of all pregnant women in Europe experience preeclampsia. ${ }^{1,2}$ The incidence of pre-eclampsia in healthy primiparous women ranges from 2 to $7 \%$. Despite the significant progress achieved in perinatal medicine, pre-eclampsia is still one of the most urgent current problems in obstetrics. Nowadays patients at high risk of developing pre-eclampsia are distinguished on the basis of clinical features, the most important ones being pregestational diabetes, increased body mass index (BMI), a history of pre-eclampsia and chronic hypertension. However, these risk factors lead to the detection of only $30 \%$ of the patients who will suffer from pre-eclampsia, and are not effective in nulliparous women without risk factors in their medical history. ${ }^{3,4}$

Pre-eclampsia prediction can be made more accurate by combining biochemical and biophysical markers, 3 of which - i.e. free beta-human chorionic gonadotropin (free $\beta$-hCG), pregnancy-associated plasma protein A (PAPP-A) and placental growth factor (PlGF) - can be used in first-trimester screening studies. ${ }^{5-9}$ To increase the sensitivity of screening studies for PE using multivariate analysis, a standardized technique for measuring blood pressure in the first trimester has been included. In the mid-1990s, uterine artery flow in the second trimester was shown to be an effective method characterized by high sensitivity but low specificity. ${ }^{10}$ Some reports indicate the value of uterine artery flow assessment in the first trimester. ${ }^{11}$ Integrated screening studies performed sufficiently early should allow the detection of patients at increased risk of pre-eclampsia.

The early identification of patients at increased risk of developing pre-eclampsia is important for several reasons. Firstly, the effectiveness of preventive interventions is dependent on the early introduction of treatment modifying the placentation process. ${ }^{3}$ Secondly, early risk stratification allows patients to be correctly qualified into groups, and for survival rates to be increased through interventions in the high-risk group. Finally, accurate predictions will improve the quality of studies evaluating the potential role of preventive actions and the pathogenesis of pre-eclampsia.

The aim of the study was to establish diagnostic algorithms for early and late pre-eclampsia and intrauterine growth restriction.

\section{Material and methods}

The study population consisted of 320 pregnant women between $11+0$ and $13+6$ weeks of gestation between 2011 and 2015. The study was performed at the Perinatology and Gynecology Department of Polish Mother's Memorial Hospital Research Institute, a tertiary care center, and in two private practices: NZOZ Medyk W. Litwiński
(Włocławek, Poland) and NZOZ Sonomedica (Łódź, Poland). The group consisted of 240 participants without risk factors in their medical history and 80 with at least one risk factor for developing pre-eclampsia (preeclampsia in a previous pregnancy, chronic hypertension, elevated BMI, kidney diseases, diabetes, systemic diseases). Women with multiple pregnancies, those with the presence of significant fetal abnormalities and those who had experienced an abortion or termination of pregnancy were excluded from the present study. All the participants in the study signed a consent form, and the approval of the Hospital Ethics Committee to conduct the study was obtained.

The presence of the risk factors listed above did not lead to pre-eclampsia in every case. Based on the actual occurrence of pre-eclampsia, the study group was subdivided into 4 groups: 1) patients who did not suffer from pre-eclampsia (the control group; $n=269$ ); 2) patients who suffered from the early form of pre-eclampsia ( $\mathrm{n}=22)$; 3) patients who developed late-onset pre-eclampsia ( $\mathrm{n}=29$ ); 4) patients diagnosed with intrauterine growth restriction $(n=43)$. An analysis of potential risk markers in these 3 groups was carried out.

An accurate interview with each patient was conducted during the first antenatal visit. The following data were recorded: age, height, parity, smoking during pregnancy, type of conception, family history (including pre-eclampsia affecting mothers), elevated blood pressure before pregnancy, diabetes, pre-eclampsia in a previous pregnancy, systemic diseases, kidney disorders or antiphospholipid syndrome. In addition, the mean blood pressure was measured, a $5 \mathrm{~mL}$ blood sample was collected and an ultrasound scan was performed. During the ultrasound examination, the following measurements were recorded, in accordance with the Fetal Medicine Foundation guidelines: the crownrump length, nuchal translucency, and the flow through the ductus venosus and uterine artery. An initial evaluation of the fetal anatomy was also performed.

The following visit, which took place between $20+0$ and $23+6$ weeks of gestation, included blood pressure measurement and an ultrasound scan, with an evaluation of fetal anatomy and growth to detect congenital fetal anomalies. Additionally, uterine artery flow was measured.

During the last visit, scheduled between $30+0$ and $34+6$ weeks of gestation, blood pressure was measured and an ultrasound scan was performed in order to evaluate the anatomy, Doppler flows and growth of the fetus.

The $5 \mathrm{~mL}$ blood samples taken during the first visit were collected in plastic tubes with ethylenediaminetetraacetic acid (EDTA). Following centrifugation, the serum was stored at $-80^{\circ} \mathrm{C}$. PlGF, PAPP-A and free $\beta$-hCG concentrations were evaluated using equipment that provides reproducible results (Delfia Xpress System, PerkinElmer, Waltham, USA).

Blood pressure was measured using equipment certified by the Fetal Medicine Foundation (3BT0-A2, Micro- 
life, Taipei, Taiwan). The devices were calibrated before and at regular intervals during the study. The measurements were performed by appropriately trained doctors. The subjects were in a seated position with their arms at the level of the heart. An appropriate adult cuff size was selected for the participant's arm circumference (small: $<22 \mathrm{~cm}$, average: $22-32$ or large: $33-42 \mathrm{~cm}$ ). After $5 \mathrm{~min}$ rest, the blood pressure was measured in both arms simultaneously at 1-min intervals until the differences between successive measurements fell within $10 \mathrm{~mm} \mathrm{Hg}$ in systolic blood pressure and $6 \mathrm{mmHg}$ in diastolic blood pressure in both arms. When stability was reached, the mean arterial pressure (MAP) of each arm was calculated as the average of the previous 2 stable measurements. The final result was taken from the arm with the highest MAP.

All the scans were performed transabdominally with a Voluson E6 and E8 ultrasound machine scanner (GE Healthcare, Chicago, USA). All the ultrasound and Doppler examinations were performed by doctors certified by the Fetal Medicine Foundation. The measurements were taken in accordance with Fetal Medicine Foundation guidelines. Pulsed wave Doppler was used to assess the uterine artery pulsatility index (UtA-PI). First, a sagittal section of the uterus was obtained and the cervical canal was identified. The transducer was then carefully tilted from side to side and color flow mapping was used to visualize each uterine artery. Pulsed wave Doppler was used with the gate set at $2 \mathrm{~mm}$ and an angle of insonation less than 30 degrees. After obtaining 3 similar consecutive waveforms, the PI was measured and the mean PI of the left and right arteries was calculated. The uterine artery pulsatility index measurements were taken in a similar way in the second trimester. The findings were collected and the risk of pre-eclampsia was assessed.

\section{Statistical analysis}

The mean PI, MAP, PlGF, PAPP-A and free $\beta$-hCG of the uterine artery were converted into multiples of median (MoM) and corrected for the crown-rump length of the fetus (CRL), the patient's age, BMI, smoking, parity, type of conception and racial origin. The distributions of the examined parameters were transformed into a Gaussian distribution after logarithmic transformation.

The measurements taken from the pre-eclampsia, IUGR and unaffected groups were compared. The comparison between the early pre-eclampsia, late pre-eclampsia and IUGR groups and the unaffected control group was made using the Mann-Whitney U test with post-hoc Bonferroni correction was used (the critical value for statistical significance was $\mathrm{p}<0.0167)$. A p-value $<0.05$ was considered significant.

A multivariable stepwise logistic regression analysis was carried out to calculate the risks of IUGR, early and lateonset pre-eclampsia based on a combination of maternal risk factors, biochemical markers (PlGF, PAPP-A, free $\beta$-hCG concentrations) and biophysical markers (MAP, UtA-PI). The performance of the screening was described by receiver-operating characteristics (ROC) curves, areas under the curve (AUC), confidence intervals (CI 95\%), sensitivity, specificity and detection rates, with a fixed false-positive rate of $10 \%$. Prediction models were developed using multivariable logistic regression analysis consisting of the following components: univariate analysis, linear examination of predictors, an examination of the correlation between predictors, an evaluation of significant interaction between potential predictors, residual analysis and model evaluation. The p-value needed to be included in the logistic regression analysis was fixed at $<0.05$. The analysis results were presented using odds, Nagelkerke's R2 and p-values.

The statistical analysis was conducted using $\mathrm{R}$ 3.1.1 software.

\section{Results}

The participants' characteristics are presented in Table 1 . The early PE group was characterized by a higher BMI, a higher incidence of chronic HA, diabetes and kidney disease than the controls. The late PE group was characterized by a higher prevalence of smokers, women with a history of pre-eclampsia and chronic hypertension than the controls. In the IUGR group, the women were found to have a higher BMI, and more often suffered from diabetes, chronic hypertension and kidney disease than the controls.

The impact of the following factors on the prediction of the early, late pre-eclampsia and IUGR was analyzed: UtA-PI, UtA-PI MoM, MAP, MPA MoM, PlGF concentration, PlGF MoM, PAPP-A concentration, PAPP-A MoM, free $\beta$-hCG and free $\beta$-hCG MoM. Data for each parameter are presented in Table 2 .

Multivariable stepwise logistic regression analysis was used to determine the effect of the following log-transformed factors: MPA MoM, mean UtA-PI MoM, PlGF MoM, PAPP-A MoM and free $\beta$-hCG MoM on the occurrence of early pre-eclampsia, late pre-eclampsia and intrauterine growth restriction. The results of the ROC analysis for early pre-eclampsia, late pre-eclampsia and IUGR are presented in Table 3.

The detection rates of the early pre-eclampsia, late preeclampsia and IUGR groups were also compared, with a fixed false-positive rate of $10 \%$ (Table 4 ).

The performance of the screening was described by the areas under the curve. The AUC values are described for all the parameters in Table 5 .

On the basis of the data, the best screening models for the prediction of early pre-eclampsia, late pre-eclampsia and intrauterine growth restriction were established and presented in ROC curves (Fig. 1-3). 
Table 1. Participants' characteristics

\begin{tabular}{|c|c|c|c|c|}
\hline & $\begin{array}{l}\text { Control } \\
(n=269)\end{array}$ & $\begin{array}{l}\text { Early pre-eclampsia } \\
\qquad(\mathrm{n}=22)\end{array}$ & $\begin{array}{l}\text { Late pre-eclampsia } \\
\qquad(\mathrm{n}=29)\end{array}$ & $\begin{array}{l}\text { IUGR } \\
(n=43)\end{array}$ \\
\hline Age (years) & $28(25-31)$ & $29.5(26-34)^{\text {NS }}$ & $30(27-33)^{N S}$ & $29(26-34)^{N S}$ \\
\hline BMI $\left(\mathrm{kg} / \mathrm{m}^{2}\right)$ & $23.9(22-27.6)$ & $30.6(23.1-32.3)$ * & $26.5(23.6-29.7) \mathrm{NS}$ & $28.7(23.6-32.4) \neq$ \\
\hline Smoking & $21(8 \%)$ & $2(9 \%)^{\text {NS }}$ & $6(21 \%)^{N S}$ & $6(14 \%)^{N S}$ \\
\hline $\begin{array}{l}\text { Parity } \\
\text { multiparous } \\
\text { primiparous }\end{array}$ & $\begin{array}{l}115(43 \%) \\
154(41 \%)\end{array}$ & $\begin{array}{r}8(36 \%)^{\text {NS }} \\
14(64 \%)^{\text {NS }}\end{array}$ & $\begin{array}{c}23(79 \%) \ddagger \\
6(21 \%) \dagger\end{array}$ & $\begin{array}{l}21(49 \%)^{N S} \\
22(51 \%)^{N S}\end{array}$ \\
\hline Preterm delivery & $13(5 \%)$ & $5(23 \%) *$ & $14(48 \%) \neq$ & $11(26 \%)^{N S}$ \\
\hline Miscarriage in medical history & $32(12 \%)$ & $6(27 \%)^{N S}$ & $6(21 \%)^{N S}$ & $17(40 \%) \neq$ \\
\hline History of pre-eclampsia & $27(10 \%)$ & $7(31 \%) *$ & $18(62 \%) \neq$ & $13(30 \%)^{N S}$ \\
\hline Diabetes & $13(5 \%)$ & $5(23 \%) *$ & $1(3,45)^{\mathrm{NS}}$ & $8(18 \%) *$ \\
\hline Chronic hypertension & $30(11 \%)$ & $12(55 \%) \neq$ & $9(31 \%) *$ & $25(58 \%) \neq$ \\
\hline Kidney disease & $5(2 \%)$ & $6(27 \%) \neq$ & $2(7 \%)^{N S}$ & $9(21 \%) \neq$ \\
\hline Pre-eclapsia in mother & $19(7 \%)$ & $2(9 \%)^{N S}$ & $3(10 \%)$ NS & $5(12 \%)^{N S}$ \\
\hline AFS & 0 & 0 & 0 & 0 \\
\hline
\end{tabular}

Data are presented as n (percent) or median (first quartile-third quartile). Comparison between groups (early pre-eclampsia, late pre-eclampsia, IUGR) and the group of patients who didn't suffer from pre-eclampsia was made for dichotomous variables using the $\chi^{2}$ test with appropriate corrections and for continuous variables using the Mann-Whitney $U$ test. For both tests post-hoc Bonferroni correction was used (the critical value for statistical significance was $p<0.0167)$. NS $p>0.0167 ;{ }^{*} p<0.0167 ; \dagger p<0.001 ; \neq p<0.0001$.

Fig. 1. Receiver-operating characteristics $(\mathrm{ROC})$ curves of maternal risk factors $+\log _{10}$ PIGF MoM ( $\cdots$ ), maternal risk factors + Log 10 UtA-PI MoM (- - ) and maternal risk factors + $\log _{10}$ PIGF MoM + $\log _{10}$ UtA-PI MoM (-) in the prediction of early-preeclampsia

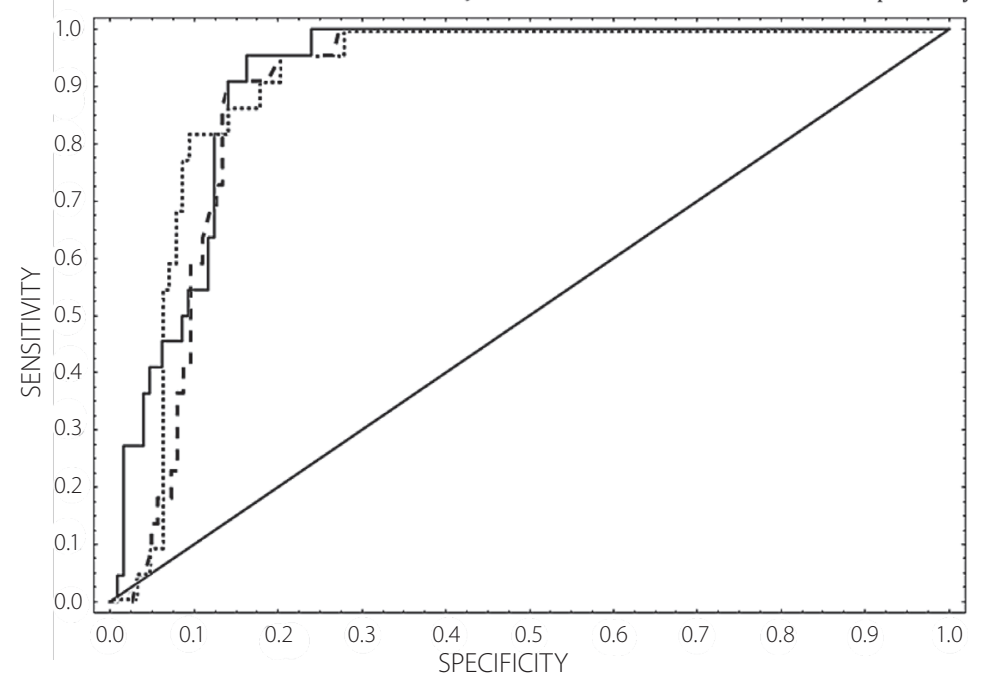

Maternal risk factors $+\log _{10}$ mean UtA PI MoM

$Y=-3.15\left(\right.$ SE 0.63) + 6.8 (SE 2.88) $\times \log _{10}$ mean UtA PI MoM + 1.8 (SE 0.52) x maternal risk factors.

Odds $=e^{Y} ; R^{2}=0.33 ; p<0.0001$.

Maternal risk factors + Log 10 PIGF MoM

$Y=-2.6$ (SE 0.55) - 2.25 (SE 1.1) x Log ᄀ10 PIGF MoM + + 1.9 (SE 0.52) x maternal risk factors.

Odds $=e^{Y} ; R^{2}=0.40 ; p<0.0001$.

Maternal risk factors + + Log 10 PIGF MoM + Log 10 śr Uta PI MoM

$Y=-3.41$ (SE 0.69) - 2.6 (SE 1.2) x $\log _{10}$ PIGF MoM + 7.09 (SE 2.88) x $\log _{10}$ mean UtA PI MoM + 1.7 (SE 0.53) x maternal risk factors.

Odds $=e^{Y} ; R^{2}=0.43 ; p<0.0001$ 
Table 2. Data for each marker in the four outcome groups

\begin{tabular}{|c|c|c|c|c|}
\hline & $\begin{array}{l}\text { Control } \\
(n=269)\end{array}$ & $\begin{array}{l}\text { Early pre-eclampsia } \\
\qquad(\mathrm{n}=22)\end{array}$ & $\begin{array}{l}\text { Late pre-eclampsia } \\
\qquad(\mathrm{n}=29)\end{array}$ & $\begin{array}{l}\text { IUGR } \\
(n=43)\end{array}$ \\
\hline Gestational age according to CRL (hbd) & $12.5(12.2-13)$ & $12.3(12-13)^{N S}$ & $12.6(12.4-13)$ NS & $12.4(12.1-13)$ NS \\
\hline $\mathrm{CRL}(\mathrm{mm})$ & $63.6(59.1-68.3)$ & $60(54.7-66.7)^{\mathrm{NS}}$ & $64.7(61.2-67.1)^{\mathrm{NS}}$ & $61.5(56.2-67.1)^{\mathrm{NS}}$ \\
\hline DVPI & $1.01(0.91-1.11)$ & $0.95(0.86-1.02)^{N S}$ & $1.01(0.92-1.05)^{\mathrm{NS}}$ & $0.96(0.91-1.04)^{N S}$ \\
\hline $\begin{array}{l}\text { Mean UtA-PI } \\
\text { Mean UtA-PI MoM }\end{array}$ & $\begin{array}{l}1.68(1.32-2.09) \\
1.07(0.86-1.29)\end{array}$ & $\begin{array}{l}2.28(2.1-2.56) \neq \\
1.42(1.26-1.62) \neq\end{array}$ & $\begin{array}{l}2.18(2.07-2.33) \neq \\
1.4(1.3-1.47) \neq\end{array}$ & $\begin{array}{l}2.33(2.12-2.62) \neq \\
1.44(1.3-1.73) \neq\end{array}$ \\
\hline $\begin{array}{l}\text { PIGF concentrations }(\mathrm{pg} / \mathrm{mL}) \\
\text { PIGF MoM }\end{array}$ & $\begin{array}{l}27.4(19.6-34) \\
1.21(0.93-1.57)\end{array}$ & $\begin{array}{l}15.55(11.1-19) \neq \\
0.62(0.51-0.96) \neq\end{array}$ & $\begin{array}{r}21(16.7-25.6) * \\
0.92(0.63-1.09) \neq\end{array}$ & $\begin{array}{l}20.5(14.6-26.2) * \\
0.97(0.59-1.12) \neq\end{array}$ \\
\hline $\begin{array}{l}\text { Free beta-hCG concentrations (IU/L) } \\
\text { Free beta-hCG MoM }\end{array}$ & $\begin{array}{l}42.1(31.2-58) \\
1.14(0.75-1.49)\end{array}$ & $\begin{array}{l}41.65(25.7-47)^{\mathrm{NS}} \\
1.08(0.74-1.23)^{\mathrm{NS}}\end{array}$ & $\begin{array}{r}42(34.1-55.8)^{\mathrm{NS}} \\
1.25(1.05-1.49)^{\mathrm{NS}}\end{array}$ & $\begin{array}{l}40.3(32.4-44.4)^{\mathrm{NS}} \\
1.12(0.91-1.25)^{\mathrm{NS}}\end{array}$ \\
\hline $\begin{array}{l}\text { Mean MAP } \\
\text { Mean MAP MoM }\end{array}$ & $\begin{array}{l}84.6(78.4-92.6) \\
0.99(0.93-1.06)\end{array}$ & $\begin{array}{c}100.35(93.1-103.7) \neq \\
1.15(1.06-1.17) \neq\end{array}$ & $\begin{array}{l}96.4(92.1-103.6) \neq \\
1.09(1.06-1.16) \neq\end{array}$ & $\begin{array}{l}95.6(90.2-102.2) \neq \\
1.12(1.03-1.16) \neq\end{array}$ \\
\hline Age at delivery (hbd) & $39(37-40)$ & $30(29-32) \neq$ & $37(35-37) \neq$ & $32(29-34) \neq$ \\
\hline Birth weight (g) & $3450(3050-3780)$ & $1015(850-1200) \neq$ & $2920(2450-3120) \neq$ & $1297(890-1760) \neq$ \\
\hline Mean UtA-PI (secondtrymester) & $1(0.82-1.32)$ & $1.63(1.45-1.9) \neq$ & $1.74(1.42-1.88) \neq$ & $1.62(1.43-1.78) \neq$ \\
\hline
\end{tabular}

Data is shown as a median (interquartile range). Comparison between groups (early pre-eclampsia, late pre-eclampsia, IUGR) and group of patients who didn't suffer from pre-eclampsia was made using $U$ Mann-Whitney test. The post-hoc Bonferroni correction was used (critical value for statistical significance was $p<0.0167)$. NS $p>0.0167 ;{ }^{*} p<0.0167 ; \uparrow p<0.001 ; \neq p<0.0001$.

Fig. 2. Receiver-operating characteristics (ROC) curves of maternal risk factors $+\log _{10}$ MAP MoM (-) and maternal risk factors + $\log _{10}$ UtA-PI MoM (- - ) in the prediction of late pre-eclampsia

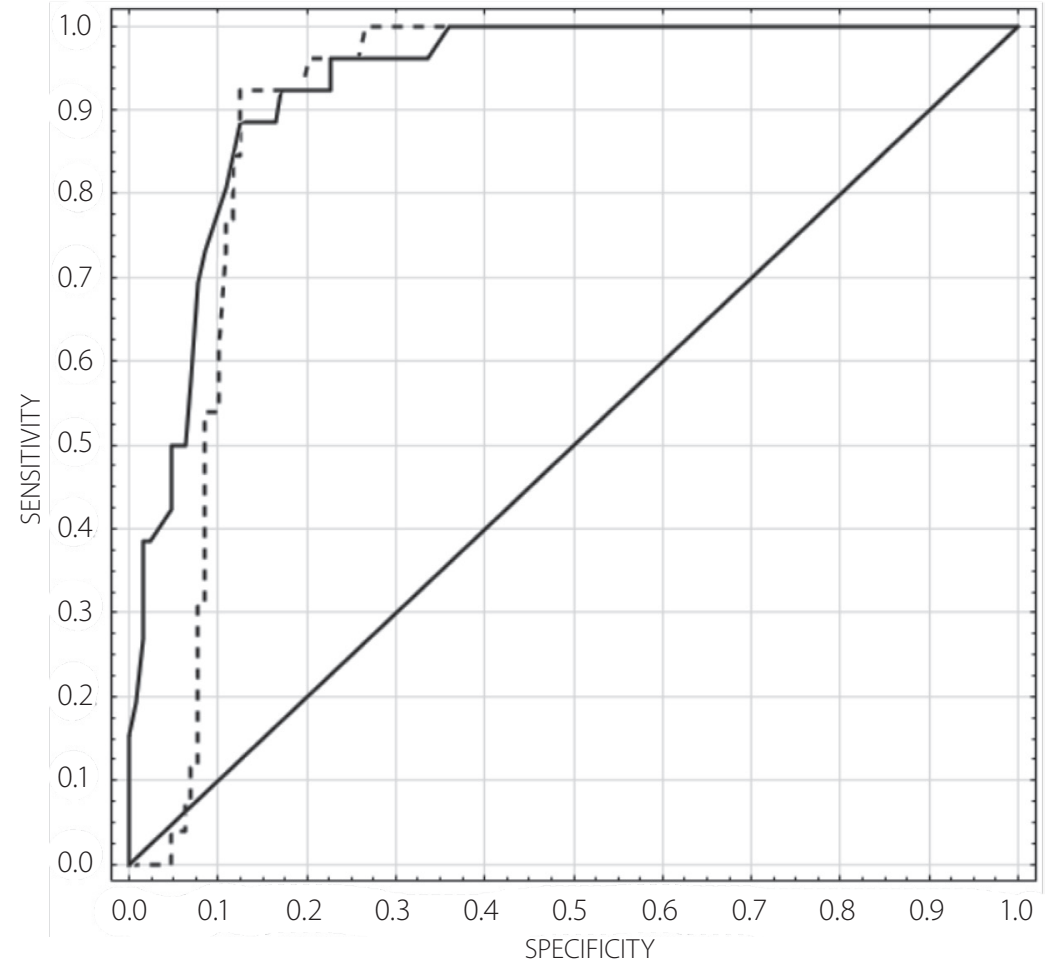

Maternal risk factors + Log $\neg 10$ mean UtA PI MoM $Y=-3.046$ (SE 0.64) + 7.058 (SE 3.02) x $\log _{10}$ Śr Uta PI $\mathrm{MoM}+1.94$ (SE 0.52) $\times$ maternal risk factors

Odds $=e^{Y}$

$\mathrm{R}^{2}=0.26$

$p<0.05$

Maternal risk factors $+\log \neg 10$ mean MAP MoM $Y=-2.204\left(\right.$ SE 0.368) $+22.75\left(\right.$ SE 7.463) $\times$ Log $_{10}$ mean MPA MoM + 1.205 (SE 0.297) $\times$ maternal risk factors Odds $=e^{Y}$

$R^{2}=0.29$

$\mathrm{p}<0.001$ 


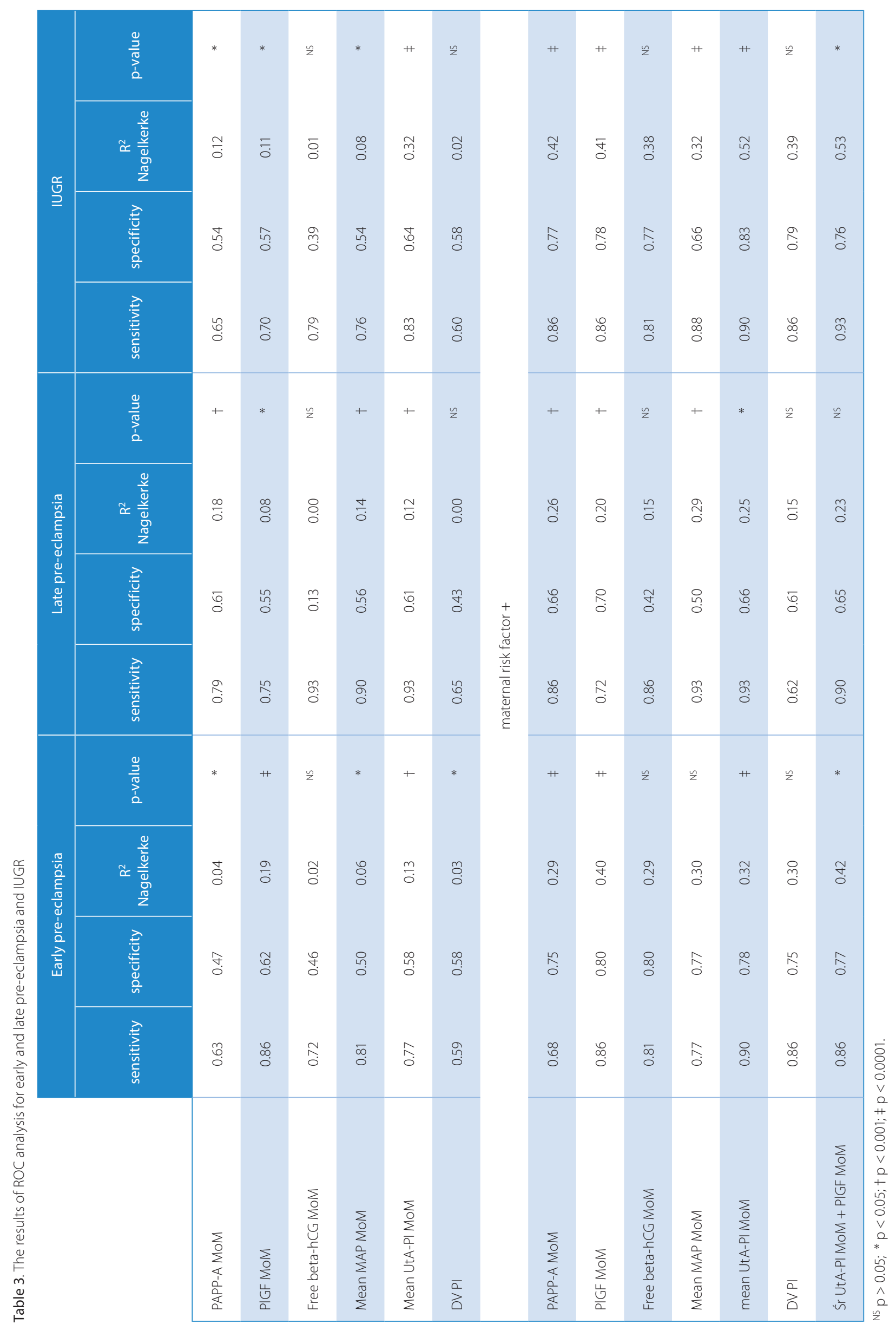




\section{Discussion}

Pre-eclampsia occurs in $2-3 \%$ of all pregnancies and is the main cause of maternal and fetal morbidity and mortality. ${ }^{1}$ The diagnosis is based on clinical features such as high blood pressure and proteinuria, which are the final steps in the pathogenesis of pre-eclampsia, beginning with impaired implantation and growth of trophoblasts in the first trimester of pregnancy. Despite the lack of acknowledged methods of prevention, 2 important benefits can be gained from defining a group of patients who may be found to be at increased risk of pre-eclampsia during routine examinations performed in the first trimester of pregnancy. Firstly, greater obstetric supervision can be provided for high risk patients, and secondly, an adequate response can be prepared in case of complications. The present analysis was conducted on a group of patients undergoing a routine scan in the first trimester of pregnancy in order to calculate the risk of chromosomal abnormalities and pre-eclampsia and its complications. The results of the statistical analysis indicate that multivariable screening based on the parameters investigated in this study allow the majority of pregnancies complicated by pre-eclampsia to be identified. This screening is more valuable in detecting early-onset pre-eclampsia than late-onset pre-eclampsia, with the respective sensitivities being 91 and $85 \%$, while the respective specificities are 84 and $83 \%$.

The combination of a detailed medical history with a blood pressure examination is the cheapest and the

Fig. 3. Receiver-operating characteristics (ROC) curves of maternal risk factors $+\log _{10}$ PIGF MoM ( $\cdots$ ), maternal risk factors + $\log _{10}$ MAP MoM (---) and maternal risk factors + $\log _{10}$ PIGF MoM + $\log _{10}$ UtA-PI MoM (-) in the prediction of intrauterine growth restriction

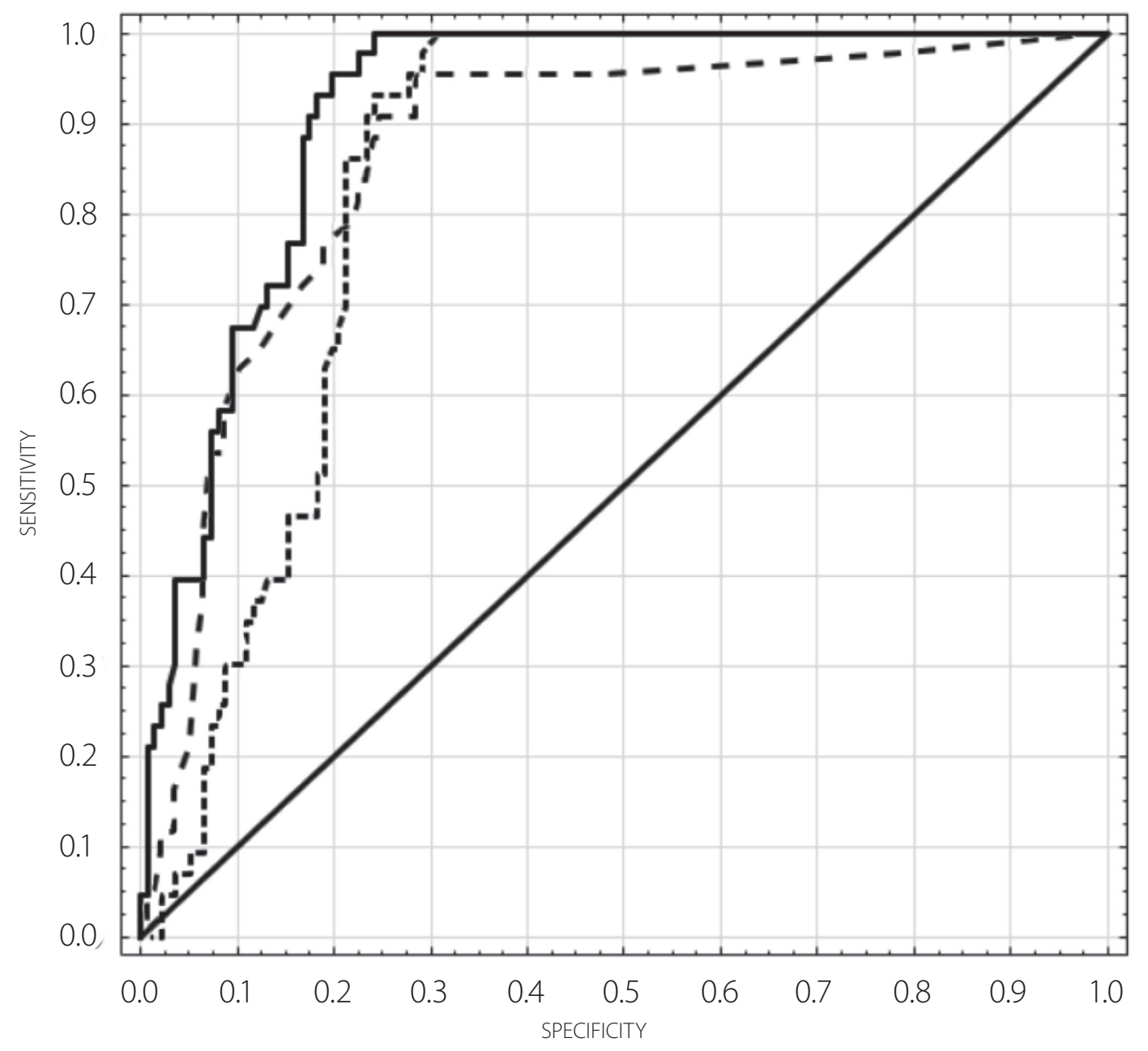

Maternal risk factors $+\log _{10}$ PIGF MoM + mean UtA PI MoM

$Y=-7.516\left(\right.$ SE 1.80) - $0.123\left(\right.$ SE 0.573) $\times$ Log $_{10}$ PIGF MoM + 4.213 (SE 1.04) x Log 10 Śr Uta PI MoM + 1.962 (SE 0.43) x maternal risk factors Odds $=e^{Y} ; R^{2}=0.54 ; p<0.05$ 
Table 4. The performance of the screening for pre-eclampsia and intrauterine growth restriction for each parameter separately and for a combination of maternal factors with biochemistry, mean UtA-PI and MAP, shown as the detection rate for a fixed false-positive rate (FPR)

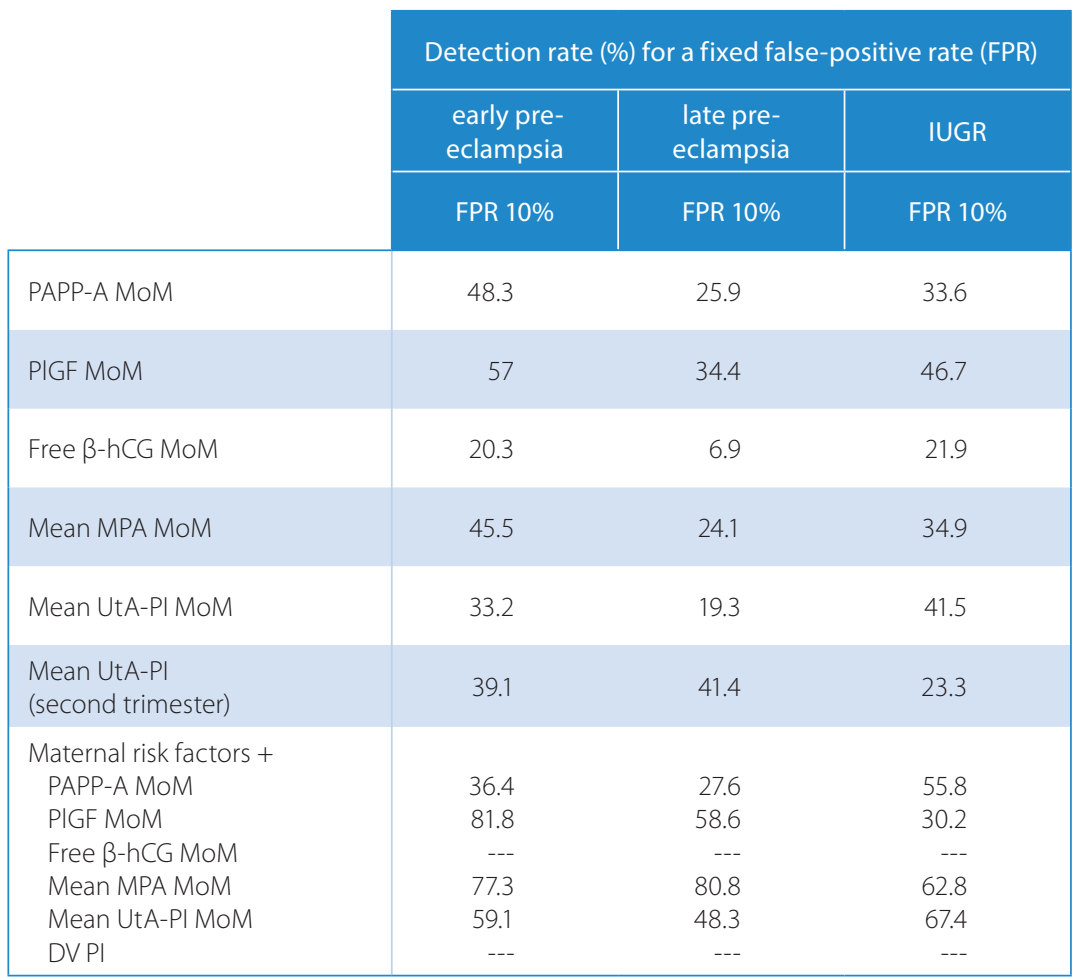

An understanding of the pathophysiology of pre-eclampsia would allow the factors participating in early angiogenesis and placentation to be evaluated as potential biochemical markers. The PAPP-A and free $\beta$-hCG examined in the present study are components of screening tests for chromosomal abnormalities. Placental growth factor - one of the factors playing a role in placentation - was found to be present in similar amounts to those identified in previous studies. ${ }^{15-17}$ The same studies also reported a decrease in PAPP-A levels between $11+0$ and $13+6$ weeks of gestation in patients who developed pre-eclampsia, after excluding pregnancies complicated by chromosomal abnormalities. ${ }^{15-17}$ Additionally, the present study found the level of PAPP-A to be significantly lower in early-onset pre-eclampsia, and a significant relationship was noted between the level of PAPP-A and the uterine artery pulsatility index. These findings are consistent with those of Poon et al. and Spencer et al., who showed the value of PAPP-A measurement in the prediction of early pre-eclampsia. ${ }^{18-20}$ Pregnancies

most readily available screening method. In the present analysis, screening tests based only on medical history were found to have a sensitivity of $41 \%$ and specificity of 95\% (RR 3.42), which is consistent with previous analyses. According to Poon et al., 37\% of patients who will develop early-onset pre-eclampsia and $29 \%$ of patients who will develop late-onset pre-eclampsia can be detected on the basis of medical history alone, albeit with a $5 \%$ false positive rate. ${ }^{3}$ If blood pressure is also measured during the examination, the area of possible pregnancy complications is expanded. The calculated detection rates for early pre-eclapsia, late pre-eclapsia and intrauterine growth restriction based only on blood pressure measurement are 45,24 and $35 \%$ respectively, with a $10 \%$ false positive rate. These results are consistent with those of Manten et al., who observed higher blood pressure in patients with a history of pre-eclampsia, even when no pre-eclampsia occurred. These patients are at increased risk of developing chronic hypertension. ${ }^{12}$

Indirect evidence of impaired placenta perfusion in preeclampsia complicated pregnancies has been revealed by Doppler studies. An increase in the uterine artery pulsatility index in the first and second trimesters is observed in patients with pre-eclampsia, particularly in early onset type and IUGR. According to Plasencia et al., detection rates in early and late-onset pre-eclampsia based on the uterine artery pulsatility index were higher in the second trimester than in the first. ${ }^{13,14}$ complicated by pre-eclampsia revealed lower levels of the markers and mediators of endothelial cell dysfunction as well as placental growth factor. A relationship was also observed between PlGF levels and the chance of developing early-onset pre-eclampsia, as previously noted by Akolekar et al. and Erez et al. ${ }^{21,22}$ In the present study, no relationship was observed between the level of free b-hCG and the occurrence of pre-eclampsia or its complications. These results are consistent with those of numerous studies conducted on large cohorts of patients. ${ }^{16,23}$

However, detection rates for early pre-eclampsia, late pre-eclampsia and IUGR calculated separately for each parameter were disappointing. The respective detection rates for early pre-eclampsia, late pre-eclampsia and IUGR, calculated on the basis of multiples of the median, were 45, 24 and 35\% for mean arterial pressure; 33, 19 and $41 \%$ for mean uterine artery pulsatility index; 57 , 34 and 47\% for PlGF concentration; and 48, 26 and 34\% for PAPP-A concentration. The false positive rate was $10 \%$. As a significant relationship was found between individual, biochemical and biophysical parameters, the next step was to create an integrated model of screening tests for pre-eclampsia - an approach analogous to the concept of early multifactorial screening for chromosomal abnormalities. The application of an integrated model of screening resulted in a spectacular improvement in the sensitivity of the test, especially regarding the early form of pre-eclampsia. Finally, after taking all 
Table 5. Comparative evaluation of screening examinations for the prediction of early and late pre-eclampsia for maternal risk factors and maternal risk factors + additional factors

\begin{tabular}{|c|c|c|c|}
\hline & \multicolumn{3}{|c|}{ Area under curve (AUC) } \\
\hline & early pre-eclampsia & late pre-eclampsia & IUGR \\
\hline PAPP-A MOM & $0.62(0.57-0.67)^{*}$ & $0.74(0.70-0.78) \dagger$ & $0.69(0.64-0.74) *$ \\
\hline PIGF MoM & $0.79(0.75-0.83) \neq$ & $0.66(0.61-0.71)^{*}$ & $0.68(0.64-0.72)^{*}$ \\
\hline Free beta-hCG MoM & $0.59(0.53-0.65)^{\mathrm{NS}}$ & $0.61(0.56-0.66)^{\mathrm{NS}}$ & $0.55(0.50-0.60)^{\mathrm{NS}}$ \\
\hline Mean MAP MoM & $0.77(0.71-0.83)^{*}$ & $0.74(0.69-0.79) \dagger$ & $0.73(0.68-0.78)^{*}$ \\
\hline Mean UtA-PI MoM & $0.75(0.71-0.79) \dagger$ & $0.75(0.71-0.79) \dagger$ & $0.82(0.79-0.85) \neq$ \\
\hline DVPI & $0.64(0.58-0.70)^{*}$ & $0.51(0.46-0.56) \mathrm{NS}$ & $0.61(0.56-0.66)^{\mathrm{NS}}$ \\
\hline Maternal risk factors & $0.85(0.81-0.89) \neq$ & $0.80(0.76-0.84) \neq$ & $0.83(0.8-0.86) \neq$ \\
\hline $\begin{array}{l}\text { Maternal risk factors + } \\
\text { PAPP-A MoM } \\
\text { PIGF MoM } \\
\text { beta-hCG MoM } \\
\text { Mean MAP MoM } \\
\text { Mean UtA-PI MoM } \\
\text { DV PI } \\
\text { Śr UtA-PI MoM + PIGF MoM }\end{array}$ & $\begin{array}{l}0.85(0.82-0.88) \neq \\
0.91(0.89-0.93) \neq \\
0.81(0.76-0.86)^{\mathrm{NS}} \\
0.92(0.89-0.95)^{\mathrm{NS}} \\
0.90(0.87-0.93) \neq \\
0.70(0.66-0.76)^{\mathrm{NS}} \\
0.92(0.90-0.94) \neq\end{array}$ & $\begin{array}{l}0.84(0.81-0.87) \dagger \\
0.86(0.83-0.89) \dagger \\
0.55(0.49-0.61)^{\text {NS }} \\
0.93(0.91-0.95) \dagger \\
0.87(0.84-0.90)^{*} \\
0.67(0.63-0.71)^{\text {NS }} \\
0.80(0.77-0.83)^{\text {NS }}\end{array}$ & $\begin{array}{l}0.85(0.82-0.88) \neq \\
0.84(0.81-0.87) \neq \\
0.81(0.77-0.85)^{N S} \\
0.86(0.83-0.89) \neq \\
0.91(0.89-0.93) \neq \\
0.73(0.69-0.77)^{N S} \\
0.91(0.89-0.93)^{*}\end{array}$ \\
\hline
\end{tabular}

CI 95\% for AUC is presented in brackets; ${ }^{\text {NS }} p>0.05 ;{ }^{*} p<0.05 ; \uparrow p<0.001 ; \neq p<0.0001$.

the parameters into account (i.e. personal history, mean arterial pressure, mean uterine artery pulsatility index, PIGF and PAPP-A concentration), the best screening model for early pre-eclampsia prediction was found to be based on a combination of maternal characteristics, the mean uterine artery pulsatility index and the placental growth factor concentration. The sensitivity and specificity of this model are 91 and $84 \%$ respectively. The best model for screening for IUGR is based on the same 3 factors, which may be related to the frequent coincidence of IUGR and early pre-eclampsia. The sensitivity and specificity of this model are 91 and $83 \%$ respectively. The combination of maternal risk factors and mean arterial pressure is the best screening tool for the prediction of late-onset pre-eclampsia, with sensitivity and specificity of 85 and $83 \%$ respectively. Early pre-eclampsia screening is particularly important because of the rapid course of the condition, the high risk of maternal and neonatal mortality and morbidity, as well as its short- and long-term maternal complications.

The main advantages of the present study are the inclusion of a large number of patients with risk factors at accurately specified gestational ages and clear criteria of pre-eclampsia. In addition, a higher detection rate was achieved by considering a variety of biochemical and biophysical markers (blood pressure measurement and Doppler studies of uterine arteries) along with a medical history taken at an accurately specified gestational age. However, a potential limitation of this study was the large inter-patient variation among the risk factors, which made it difficult to obtain statistical significance for some parameters.

The ability to predict the occurrence of pre-eclampsia in early pregnancy enables maternal and fetal morbidity to be reduced through strict obstetric surveillance as well as planned delivery in a reference center. The integrated model of screening presented in this study can be valuable in defining the group of patients at increased risk of developing pre-eclampsia. Future studies are necessary on the potential role of pharmacological interventions starting in the first trimester of pregnancy with the aim of improving the quality of placentation and reducing the prevalence of pre-eclampsia.

\section{Conclusions}

Prediction of early pre-eclampsia is most effective when based on a combination of maternal risk factors, the mean uterine artery pulsatility index and placental growth factor concentration.

Prediction of late pre-eclampsia is most effective when based on a combination of maternal risk factors and mean arterial blood pressure.

Prediction of IUGR is most effective when based on a combination of maternal risk factors, the mean uterine artery pulsatility index and placental growth factor concentration. 


\section{References}

1. ACOG practice bulletin: Hypertension in pregnancy. Establishing the diagnosis of preeclamsia and eclampsia. Obstet Gynecol. 2013;17-20.

2. Di Lorenzo G, Ceccarello M, Cecotti V, et al. First trimester maternal serum PIGF, free $\beta$-hCG, PAPP-A, PP-13, uterine artery Doppler and maternal history for the prediction of preeclampsia. Placenta. 2012;33:495-501.

3. Poon LC, Kametas NA, Chelemen T, Leal A, Nicolaides KH. Maternal risk factors for hypertensive disorders in pregnancy: A multivariate approach. J Hum Hypertension. 2010;24:104-110.

4. Yu CK, Smith GC, Papageorghiou AT, Cacho AM, Nicolaides KH. An integrated model for the prediction of pre-eclampsia using maternal factors and uterine artery Doppler velocimetry in unselected low-risk women. Am J Obstet Gynecol. 2006;195(1):330.

5. Poon LC, Kametas NA, Maiz N, Akolekar R, Nicolaides KH. First-trimester prediction of hypertensive disorders in pregnancy. Hypertension. 2009;53: 812-818.

6. Leslie K, Thilaganathan B, Papageorghiou A. Early prediction and prevention of pre-eclampsia. Best Pract Res Clin Obstet Gynaecol. 2011;25:343-354.

7. Akolekar R, Syngelaki A, Poon L, Wright D, Nicolaides KH. Competing risks model in early screening for preeclampsia by biophysical and biochemical markers. Fetal Diagn Ther. 2013;33:8-15.

8. Poon LC, Akolekar R, Lachmann R, Beta J, Nicolaides KH. Hypertensive disorders in pregnancy: Screening by biophysical and biochemical markers at 11-13 weeks. Prenat Diagn. 2010;30:216-223.

9. Staff AC, Benton SJ, von Dadelszen P, et al. Redefining preeclampsia using placenta-derived biomarkers. Hypertension. 2013;61:932--942.

10. Harrington K, Carpenter RG, Goldfrad C, Campbell S. Transvaginal Doppler ultrasound of the uteroplacental circulation in the early prediction of pre-eclampsia and intrauterine growth retardation. Br J Obstet Gynaecol. 1997;104:674-681.

11. Poon LC, Stratieva V, Piras S, Piri S, Nicolaides KH. Hypertensive disorders in pregnancy: Combined screening by uterine artery Doppler, blood pressure and serum PAPP-A at 11-13 weeks. Prenat Diagn. 2010;30:216-223.

12. Manten GT, Sikkema MJ, Voorbij HA, Visser GH, Bruinse HW, Franx A. Risk factors for cardiovascular disease in women with a history of pregnancy complicated by preeclampsia or intrauterine growth restriction. Hypertens Pregnancy. 2007;26(1):39-50.
13. Plasencia W, Maiz N, Bonino S, Kaihura C, Nicolaides KH. Uterine artery Doppler at $11+0$ to $13+6$ weeks in the prediction of preeclampsia. Ultrasound Obstet Gynecol. 2007;30(5):742-749.

14. Plasencia W, Maiz N, Poon L, Yu C, Nicolaides KH. Uterine artery Doppler at $11+0$ to $13+6$ weeks and $21+0$ to $24+6$ weeks in the prediction of pre-eclampsia. Ultrasound Obstet Gynecol. 2008;32(2):138--146.

15. Ong CY, Liao AW, Spencer K, Munim S, Nicolaides KH. First trimester maternal serum free beta human chorionic gonadotrophin and pregnancy associated plasma protein A as predictors of pregnancy complications. BJOG. 2000;107(10):1265-1270.

16. Smith GC, Stenhouse EJ, Crossley JA, Aitken DA, Cameron AD, Connor JM. Early pregnancy levels of pregnancy-associated plasma protein $a$ and the risk of intrauterine growth restriction, premature birth, preeclampsia, and stillbirth. J Clin Endocrinol Metab. 2002;87(4):1762-1767.

17. Yaron Y, Heifetz S, Ochshorn Y, Lehavi O, Orr-Urtreger A. Decreased first trimester PAPP-A is a predictor of adverse pregnancy outcome. Prenat Diagn. 2002;22(9):778-782.

18. Poon LC, Maiz N, Valencia C, Plasencia W, Nicolaides KH. First-trimester maternal serum pregnancy-associated plasma proteinA and pre-eclampsia. Ultrasound Obstet Gynecol. 2009;33(1):23-33.

19. Spencer K, Cowans NJ, Chefetz I, Tal J, Meiri H. First-trimester maternal serum PP-13, PAPP-A and second-trimester uterine artery Doppler pulsatility index as markers of pre-eclampsia. Ultrasound Obstet Gynecol. 2007;29(2):128-134.

20. Poon LC, Nicolaides KH. First-trimester maternal factors and biomarker screening for preeclampsia. Prenat Diagn. 2014;25.

21. Erez O, Romero R, Espinoza J, et al. The changein concentrations of angiogenic and anti-angiogenic factors inmaternal plasma between the first and second trimesters in risk assessment for the subsequent development of preeclampsia andsmall-for-gestational age. J Matern Fetal Neonatal Med. 2008;21:279-287.

22. Akolekar R, Zaragoza E, Poon LC, Pepes S, Nicolaides KH. Maternal serum placental growth factor at $11+0$ to $13+6$ weeks of gestation in the prediction of pre-eclampsia. Ultrasound Obstet Gynecol. 2008;32(6):732-739.

23. Spencer K, Yu CK, Cowans NJ, Otigbah C, Nicolaides KH. Prediction of pregnancy complications by first-trimester maternal serum PAPP-A and free beta-hCG and with second-trimester uterine artery Doppler. Prenat Diagn. 2005;25(10):949-953. 